\title{
Téoros
}

Revue de recherche en tourisme

\section{Activités culturelles et touristiques relatives aux Amérindiens et aux Inuits}

\section{Michel Noël}

Volume 17, numéro 2, été 1998

L'industrie touristique autochtone

URI : https://id.erudit.org/iderudit/1072281ar

DOI : https://doi.org/10.7202/1072281ar

Aller au sommaire du numéro

Éditeur(s)

Université du Québec à Montréal

ISSN

0712-8657 (imprimé)

1923-2705 (numérique)

Découvrir la revue

Citer ce document

Noël, M. (1998). Activités culturelles et touristiques relatives aux Amérindiens et aux Inuits. Téoros, 17(2), 48-52. https://doi.org/10.7202/1072281ar d'utilisation que vous pouvez consulter en ligne.

https://apropos.erudit.org/fr/usagers/politique-dutilisation/ 


\section{f Canner \\ Activités culturelles et TOURISTIQUES RELATIVES AUX AMÉRINDIENS ET AUX INUITS}

\section{Michel Noël}

Le 21 juin est la journée nationale des autochtones du Canada. Elle est fêtée par toutes les communautés amérindiennes et inuit qui, pour l'occasion, organisent des manifestations culturelles haut en couleur : festival de musique, de chants, de danses, activites sportives, mise en valeur du patrimoine, vente d'artisanat, exposition d'auvres d'art, journée gastronomique, etc.

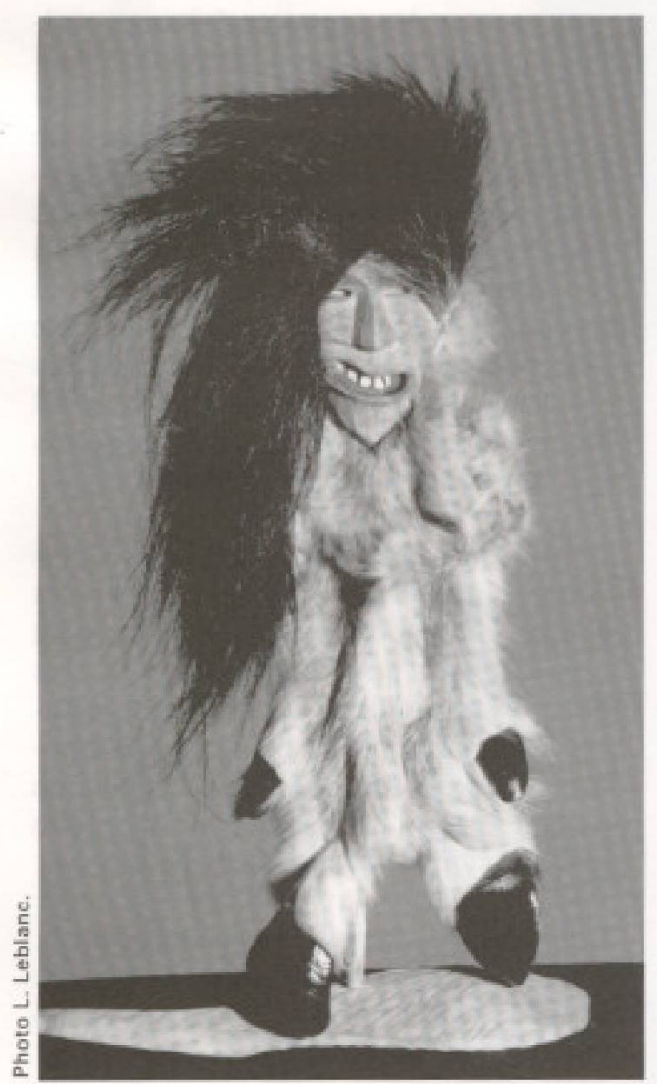

Personnage de la mythologie amerindienne, L. G. Jourdain, artiste innu.

\section{Montréal}

Festival international du film et de la vidéo autochtones ainsi que des activités culturelles et artistiques Terres en vue : (514) 521-2714

\section{Festival Innu Nikamu}

Musique autochtone traditionnelle et contemporaine Uashat-Maliotenam, au mois d'aoôt (418) $927-2985$

\section{Natashquan}

Fête du saumon,

mois de juin

(418) $726-3529$

\section{Kanesatake}

Pow-wow traditionnel

11-12 juillet

(450) $479-8881$

\section{Odanak}

Pow-wow annuel

4-5 juillet

Musée des Abénaquis

(450) $568-2600$

\section{Wemotaci}

Fète culturelle, danses,

chants, tambour

$11-13$ septembre

(819) 523-6153

\section{Atikamekw}

Nikamowin, spectacles de musiciens Atikamekw, Cris et Innu-Montagnais 14-15 août, (418) 843-3873

\section{Ouje-Bougoumou}

Festival d'été fin juillet

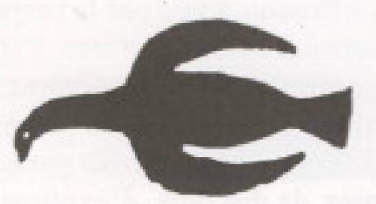

Les activités culturelles et touristiques relatives aux Amérindiens et aux Inuit sont de plus en plus nombreuses au Quebec. Elles ont lieu surtout au cours de l'été. Les musées situés en milieu urbain, rural ou même dans certaines communautés isolées, possèdent de prestigieuses collections qui mettent en valeur les richesses malheureusement méconnues des patrimoines millénaires des peuples autochtones. Heureusement, il y a un regain d'intérêt pour tout ce qui a trait a l'histoire, à la culture, au mode de vie et à l'expression artistique des descendants des " premières nations : à peupler, il y a plusieurs millénaires, le continent nord-américain. Voici une liste sommaire des musées et des lieux d'exposition les plus intéressants ainsi que des activités les plus connues. Il est à remarquer que chaque communauté autochtone du Québec possède une histoire originale et un mode de vie qui lui sont propres et que l'on y trouve diverses infrastructures d'accueil : musées, centres d'interprétation, restaurants, hôtels, pourvoiries, kiosques d'information touristique, etc. La visite la plus interessante reste cependant celle que l'on prépare à l'avance. Il est donc recommandé de contacter le Conseil de bande ou les organismes appropriés des communautés que l'on désine visiter avant de s'y rendre.

\section{TOURISME}

Aventure inuit

Voyage F. P. N. Q. inc.

19950, rue Clark-Graham

Baie d'Urfe (Québec) HgX 3R8

T6I. : (514) 457-2236

Télec. : (514) 457-4626 
Aventures Mistassini

336, boulevard Mistassini

Lac Mistassini (Québec) GoW 1 EO

Tél. : (418) 923-2580

Nessipi Kantuet

Case postale 744

Schefferville (Québec) GOG 2T0

Tel. = (418) 585-3756

Club d'aventures Naskapi

Case postale 939

Schefferville (Québec) GOG 2TO

Tél. : (514) 585-2612

Télec. : (514) 585-3953

Wawati

Case postale 118

Val d'Or (Québec) J9P 4N9

Tél. / Télec. : (514) 824-7653

Mingan

Circuit touristique montagnais

Mingan (Québec.)

Tél. : (418) 949-2234

Societé touristique Innu inc 50, boul. Bastien, local 102

Wendake, (Québec) G0A 4Vo

Tél. : (418) 843-5030

Circuits métropole Kanien

Kehaka visite peddestre de Kahnawake

Tél. : (514) 481-8092/(514) 481-7906

Les Chalets Six Saisons

Atikamekw

Ernest Ottawa

80, rue Metapeckeka

Manouane (Québec) JoK 1 Mo

Tál. : (819) $971=1455$

Télec: : $(819) 971-1484$

Camp des dix

David Marcel Ottawa

350, rue Simon-Ottawa

Manouane (Québec) JOK 1 MO

Tél. / Tólec. : 971-8819

Coocoocache

Jacques Newashish

Lucien Awashish

Wemotaci (Québec) GOX 3R0

T6́. : (514) 666-2366

Essipit

La Communauté Montagnaise

27, rue de la Réserve

Les Escoumins (Québec) GoT 1 Ko

Tél. (418) 232-6662/(418) 232-6778/

(41B) 233-2509

Auberge Kukum

241, rue Oulatchouan

Mashteuiatsh (Ouébec) GoW $2 \mathrm{HO}$

Tel. : (514) 275-0697

Télec. : (514) 275-6481

Aventure Mikuan

140, rue Oulatchouan

Mashteuiatsh (Ouébec)

T실. ; (418) 275-2949
Wigwam. La maison du tourisme

culturel en milieu autochtone

62, rue Déziel

Lévis (Québec) G6V $3 T 8$

T6́l. : (418) 833-0663

Vieux village indien de Kahnawake

Tél. : (514) 638-6521/(514) 632-7674

\section{ÉVÉNEMENTS}

Terres en vues

Festival du film et de la vidéo

Autochtone de Montréal

770, rue Rachel Est

Montréal (Québec) H2L $2 \mathrm{H}_{5}$

Tél. : (514) $521-2714$

Information sur le festival : (514) 521-5383

\section{ORGANISMES INUIT}

DE DÉVELOPPEMENT

Administration régionale Kativik

Case postale 9

Kuujiuaq (Québec) JoM 1 Co

Tếl. : (819) 964-2961

Télec. : (819) 964-2956

Fédération des cooperatives

du Nouveau-Québec

19950, rue Clark-Graham

Baie d'Urfé (Québec) H9X 3R8

Tél. : (514) 457-9371

Commission scolaire Kativik

305, rue Mimosa

Dorval (Québec) H9S 3K5

Tél. : (514) 636-8120

Conseil régional de développement

C.R.D. Kativik

Case postale 239

Kuujiuaq (Québec) JoM $1 \mathrm{Co}$

Tél. : (819) 964-2611

\section{LE NUNAVIK :}

\section{Où ? Quand? Comment ?}

II est indispensable de preparer le voyage à l'avance et de prendre contact avec les organismes appropries pour obtenir des renseignements: sur les possibilités d'hébergement et les voya. ges organisés.

Association touristique du Nunavik

Kuujjuaq (Québec)

Tél. : (819) 964-2065

Télec. : (819) 964-2611

Safari Nordik

639, boulevard Labelle

Blainville (Québec) J7C $3 \mathrm{HM}$

Tél. : (514) $971-1800$

Télec: : (514) 971-1771

Circuits été

Exploration de rivières
Federation des cooperatives du Nouveau-Québec 19950 , rue Clark-Graham

Baie d'Urfé (Québec) H9X 3R8

Tél. : (514) 457.9371

Télec. : (514) 457-4626

Institut culturel Avataq Inukjuak (Québec) JoM 1 Mo

Tól. : (819) 254-8919

Télec. : $(819)$ 254-8148

Musée

Aventures inuit

19950 , rue Clark-Graham

Baie d'Urfé (Québec) $\mathrm{HgX} 3 \mathrm{Rg}$

Tél. : (514) 457-9371

Télec. : (514) 457-4626

Circuits-aventures hiver et étê : traîneau à chiens, motoneige, safari-photo et circuits culturels.

Quasegiak Charter

Salluit (Québec) JoM 1 So

Tél. : (819) 255-8120

Télec. : (819) 255-8360

Observation de la faune

Institut culturel Avataq

650, 32" Avenue

Lachine (Québec) H8T 3 K5

Tell. : (514) 637.9887

Télec. : (514) 637-9707

Centre de documentation

\section{ORGANISATIONS POLITIQUES AUTOCHTONES ET LEURS BUREAUX RÉGIONAUX}

\section{Abénaquis}

Grand conseil de la nation Waban-Aki inc.

Siège administratif

157, rue Brassard

Case postale 430

Nicolet (Québec) JOG 1 EO

Tél. : (819) 293-6801

Télec. : (819) 293-6807

\section{Algonquins}

Secrétariat des programmes et services de la nation algonquine Réserve de Témiscamingue

Case postale 367

Notre-Dame-du-Nord (Québec) J0Z $3 B 0$

Tél. : (819) 723-2019

Télec. : (819) 723-2345

Algonquin Anishinable Nation

Case postale 313

Maniwaki (Québec) J9E $3 \mathrm{Cg}$

Tél. : (819) 449-1225

Télec. : $(819) 449-5673$

\section{Atikamekw}

Conseil de la nation atikamekw

(Atikamekw-Sipi)

317 , rue Saint-Joseph

Case postale $\mathbf{8 4 8}$

La Tuque (Québec) G9X $3 \mathrm{P} 6$

T6́l. : (819) 523-6153

Télec. : (819) 523-8706 
Grand conseil de la nation Waban-Aki inc. Siège social

58, rue Waban Aki

Odanak (Québec) JoG $1 \mathrm{Ho}$

Tél. : (514) 568-2810

\section{Cris}

Grand conseill des Cris du Québec Némiscau (Québec) J0Y 3B0

Tel. : (819) 673-2600

Grand conseil des Cris du Québec

1. Place Ville-Marie, Bureau 3438

Montréal (Québec) H3B 3N6

Tél. : (514) 861-5837

Télec. : (514) 871-0760

\section{Hurons}

Conseil de la nation huronne-wendat

225. Place Michel Laveau

Wendake (Québec) G0A 4V0

Tél. : (418) 843-3767

Tólec. : (418) 842-1108

Inuit

Société Mativik

Case postale 179

Kuujiuag (Québec) JoM $1 \mathrm{CO}$

Tél. : (819) 964-2925

Télec. : (819) 964-2613

Sociêté Mativik

$650,32^{\prime}$ avenue

Lachine (Québec) H8T 3K5

T6́l. : (514) 634-8091

Télec. : (514) 634-8091 (poste 212)

\section{Malécites}

Conseil de la nation malécite de Viger 1078, rue Sainte-Hólène

Longueuil (Québec) J4K 3R9

Tel. : (514) 674-2030

Télec. : (514) 674-2023

Miemacs

Conseil de bande des Micmacs de Gesgapegiag

Case postale 1280

Maria (Québec) GoC 1Yo

Tél. : (418) 759-3441

Télec. : (418) 759-5856

Conseil de bande de Gaspé

Case postale 69 , Fontenelle

Gaspé (Québec) GoE $1 \mathrm{Ho}$

Tél. : (418) 368-6005

Conseil de bande de Restigouche

17, rue Riverside Ouest

Restigouche (Québec) GoC 2Ro

T혜. : (418) 788-2904

Télec. : (418) 788-2058

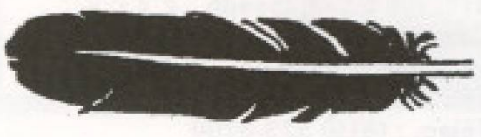

Mohawks

Conseil de bande de Kahnawake

Case postale 720

Kahnawake (Québec) JoL 1B0

Tél. : (514) 632-7500

Télec. : (514) 638-5958

Conseil des Mohawks de Kanesatake

Case postale 607

Kanesatake (Québec) JON 1 EO

Tél. : (514) 479-8373

Conseil des Mohawks d'Akwesasne

Case postale 759

Akwesasne

Via Cornwall (Ontario) K6H 5 T3

Tél. : (613) 575-2348

\section{Montagnais-Innu}

Mamit Innuat

Case postale 335

Mingan (Québec) GoG 1Vo

Tél. : (418) 949-2215

Conseil tribal Mamuitun

20, rue Messek

Betsiamites (Québec) GoH $1 \mathrm{B0}$

Tél. : (418) 567-2295

Télec. : (418) 567-8528

\section{Naskapis}

Conseil de bande de Kawawachikamach Case postale 970

Kawawachikamach (Québec) GoG 2TO

Tel. : (418) 585-2686

Télec. : (418) 585-3130

\section{Autres regroupements}

Secrétariat de l'Assemblée des Premières Nation du Québec et du Labrador 430 , rue Koska

Wendake (Québec) G0A 4V0

Tél. : (418) 842-5020/(418) 842-5274

Télec. : (418) 842-2660

Association des Femmes Autochtones du Québec

1450 , City Councillors

Bureau 440

Montréal (Québec) H3A 2E5

Tél. : (514) 844-9618/1-800-363-0322

Télec. : (514) 844.2108

Alliance Autochtone du Québec

21, rue Brodeur

Hull (Quebec) J8Y 2P6

Tél. : (819) 770-7763

Telec. : (819) 770-6070

Association des Métis et Indiens hors réserves du Québec

713, boulevard St-Joseph

Roberval (Québec) G8N 2L3

Tél. : (418) 275-0198

Télec. : (418) 275-6374

Indian Rights for Québec Indian Women Case postale 614

Kahnawake (Québec) JOL 1BO

Tell. : (514) 632-6304

\section{INSTITUTIONS CULTURELLES}

\section{Abénaquis}

Musée des Abénaquis

108, rue Waban-Aki

Okanak (Québec) JoG $1 \mathrm{Ho}$

Tél. : (514) 568-2600

\section{Algonquins}

Centre culturel et éducatif

Lac Simon

Abitibi (Québec)

Tél. : (819) 736-2351

Société Marcite8eia

10, rue Abitibiwinni

Pikogan (Québec) J9T 3A3

Tél. : (819) 732-0167

River Desert Cultural Education Centre

Bande indienne de Maniwaki

Case postale 309

Maniwaki (Québec) J9E 3C9

Tél. : (418) 449-5039

\section{Montagnais}

Institut culturel et éducatif Montagnais inc (ICEM)

1005, boul. Laure, bureau 305.2

Sept-îles (Québec) G4R 4 S6

Téí. : (418) 968-4424,1-800-391-4424

Musée amérindien de Pointe-Bleue 407, rue Amishk

Mashteuiatsh (Québec) GOW $2 \mathrm{HO}$

Tél. : (418) 275-4842

\section{Hurons}

Musée Arouänne

10, Terrain-du-Seigneur

Wendake (Québec) GOA 4 V0

Tél. : (418) 845-1241

Conseil en éducation des premières nations 240, Place Sondawka

Wendake (Québec) GOA 4V0

Tél. : (418) 842-7672

Centre culturel et éducatif

Village-des-Hurons

145, rue Aimé-Romain

Wendake (Québec) GOA 4 Vo

Tél. : (418) 843-3767

Inuit

Institut culturel Avatac

Inukjuak (Québec) JoM 1 MO

Tél. : (819) 254-8919

Musée Inuit d'Inukjuak Inukjuak (Quếbec) JoM 1 Mo Tél. : (819) 254-8919

\section{Micmacs}

Gesgapegiag Cultural Education Centre Case postale 1280 , Wesjwapniag Gesgapegiag (Québec) GOC 1 Yo Tél.: (418) 759-3422 
Restigouche Micmac Craft Association 29, Gaspe Highway

Restigouche (Québec) G0C 2R0

Tél. : (418) 788-5354

Restigouche Institute of Cultural Education 1 , rue Riverside Ouest

Restigouche (Québec) GoC 2Ro

Tél. : (418) $788-2904$

Centre d'interprétation

de la culture micmacque

Pestigouche (Québec) GOC 2RO

Tél. : (418) 788.2136

\section{CENTRES D'AMITIÉ \\ AUTOCHTONES DU QUÉBEC}

Centre d'amitié autochtone de Québec

234, rue Saint-Louis

Loretteville (Québec) G2B 1 L4

Tél. : (418) 843-5818

Télec. : (418) 843-8960

Centre d'amitié Innu inc.

Boite postale 81

Maliotenam (Québec) G4R $4 \mathrm{~K} 2$

Tél, ; (418) 968-2026

Centre d'amitié et d'entraide de la Tuque 544, rue Saint-Antoine

La Tuque (Québec) $\mathrm{GgX} 2 \mathrm{Y}_{4}$

Tél. : (819) 523-8637

Centre d'amitié autochtone de Senneterre $910,10^{\circ}$ Avenue - Case postale 1769 Senneterre (Ouébec) JoY $2 \mathrm{M0}$

Tél. : (819) 737-2324

Centre d'amitié autochtone de Montréal 3730, Cote-des-Neiges

Montréal (Québec) H3N 1 V6

Tél. : (514) 937-5338

Centre d'amitiế autochtone de $\mathrm{Val} \mathrm{d}^{\top} \mathrm{Or}$ 1011,6 . Rue

Val d'Or (Québec) J9P 3W4

Tél. : (819) B25-6958

Centre d'amitié autochtone de

Chibougamau

95, rue Jaculet

Chibougamau (Québec) G8P $2 \mathrm{G} 4$

Tél. (418) $748-2050 /(418) 748-7667$

Regroupement des centres d'amitié autochtones du Québec 30, rue de l'Ours

Wendake (Ouébec) GOA 4 VO

Tél. : (418) 842-6354

Regroupement des centres d'amitié autochtone du Québec 1011, 6* Rue

Val d'Or (Ouébec) J9P 3W4

Tél. : (819) 825-6857

\section{OUTRE-MER}

Galerie Saint-Merri

Art Inuit

9. rue Saint-Merri

75004 Paris, France

Tél. : $1-42.77 .39 .12$

Télec. : 1-40.71.02.20

D.A.Q. Mme Beneteau

93330 Neuilly-sur-Marne, France

Tél : 43.08 .94 .23

Atoka Diffusion

17. Place du Moütier

94800 Villejuif, France

Tél. : 47,26,64.81

L'Iglou Art Esquimau

35, rue N.D. des Wets

59500 Douai, France

T61. : 27.88.29.14

Inuit Galerie

Augustann Lage 3

6800 Mannheim 1. Allemagne

Tel. : $(0621) 41.49 .96$

\section{MUSÉES, CENTRES D'EXPOSITION ET LIEUX TOURISTIQUES AMÉRINDIENS ET INUIT}

\section{Montréal}

Centre d'histoire de Montréal

335. Place d'Youville

Montréal (Québec) H2Y 3T1

Tél : (514) 872-3207

Télec. : (514) 872-9645
Musée du Chäteau Ramezay

280, rue Notre-Dame Est

Montréal (Québec) H2Y $1 \mathrm{C} 5$

Tel. : (514) 861.7182

Télec. : (514) 861-8317

Musée Marguerite-Bourgeois 400 , rue Saint-Paul Est

Montréal (Québec) $\mathrm{H}_{2} \mathrm{Y} 1 \mathrm{H}_{4}$

Tél. : (514) 845.9991

Musée des Beaux-Arts de Montréal

1379, rue Sherbrooke Ouest

Case postale 3000 , Succursale H

Montréal (Québec) H3Y 2T9

Tél. : (514) 285.1600

Télec. : (514) 844-6042

Musée McCord d'histoire canadienne 690, rue Sherbrooke Ouest

Montréal (Québec) H3A 1 E9

Tél. : (514) 398.7100

Télec. : (514) 398-5045

Pointe-à-Callière, Musée d'archéologie et d'histoire de Montréal

350, Place Royale

Vieux Montréal (Québec) H2Y 3Y5

Tél. : (514) 872-9150

Télec. : (514) 872-9151

\section{Montréal (banlieue et région)}

Musée régional d'Argenteuil

50 , rue Principale

Carillon (Québec) JoV $1 \mathrm{CO}$

Tél. : (514) 537-3861

Lieu historique national du Fort Chambly 2, rue Richelieu

Chambly (Québec) J3L 2B9

Tél. : (514) 658-1585

Télec. : (514) 658-2428

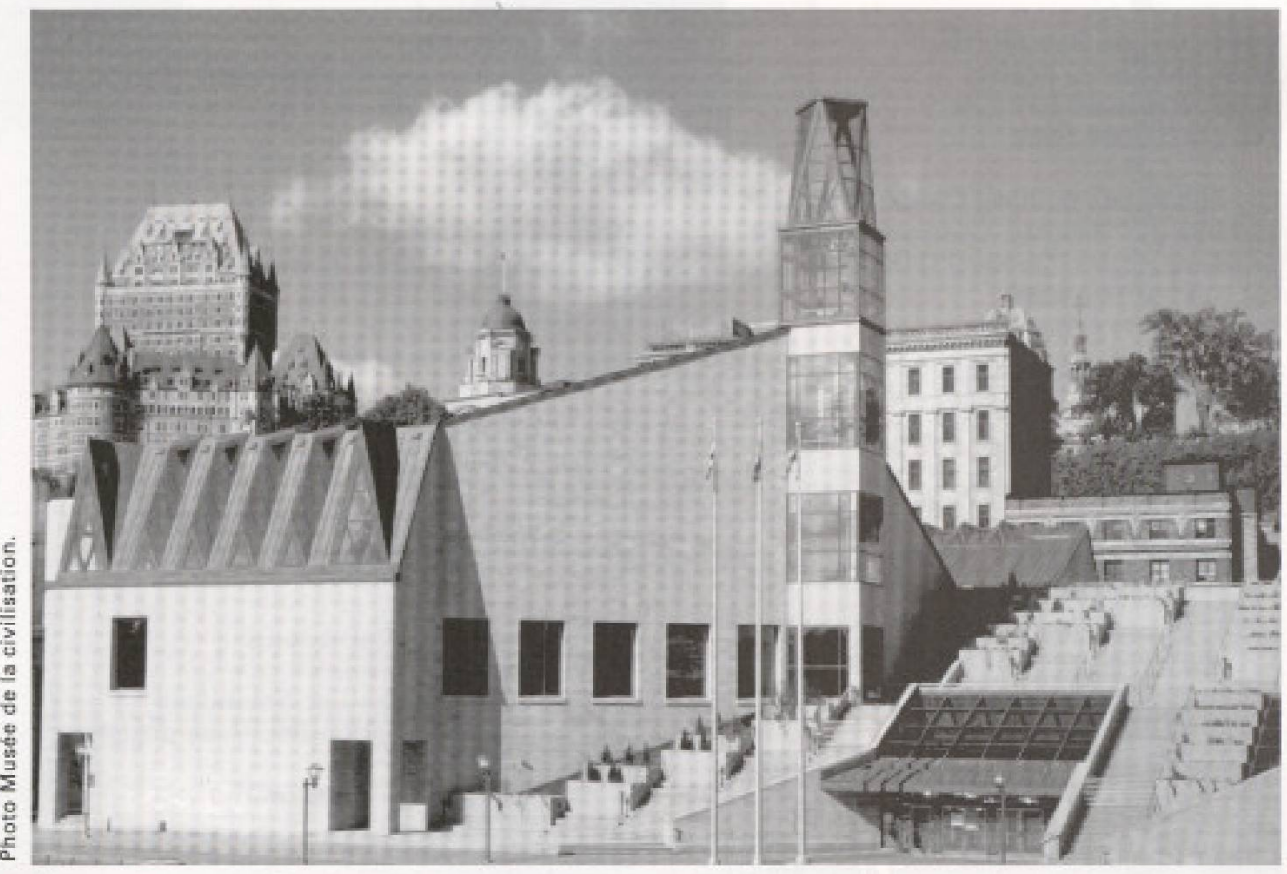

A l'automne 1998, le Musée de la civilisation, à Québec, procédera à l'ouverture d'une imposante exposition permanente sur les peuples autochtones du Québec. 
Lieu national de Côteau-du-Lac

Chemin du Fleuve, Case postale 550

Côteau-du-Lac (Québec) JoP 1 B0

Tél. : (514) 763-5631

Parc archéologique de la Pointe-du-Buisson 333, rue Émond

Melocheville (Québec) JOS 1J0

Tél. : (514) 429-7857

Télec. : (514) 429-5921

Musée régional du Haut-Richelieu

182, rue Jacques-Cartier Nord

Saint-Jean-sur-le-Richelieu (Québec) J3B

$7 \mathrm{~W} 3$

Tél. : (514) 347-0649

Télec. : (514) 347-9994

Lieu historique national du

Commerce de la fourrure à Lachine

1255, boulevard Saint-Joseph

Lachine (Québec) H8S 2M2

Tél. : (514) 637-7433

Kanien" Kehaka Raotitiohkwa Cultura

Centre

Case postale 1988

Kahnawake (Québec) JOL 1B0

Tél. : (514) 638-0880

Télec. : (514) 638-0920

\section{Québec}

Musée de la Civilisation

85, rue Dalhousie

Case postale 155, Succursale B

Québec (Québec) G1K 7A6

Tél. : (418) 643-2158

Télec. : (418) 646-9705

Musée des Ursulines de Québec

12, rue Donnacona, Case postale 760, H.V. Québec (Québec) G1R 4T1

Tél. : (418) 694-0694

Vieille Maison des Jésuites

2320, chemin du Foulon

Sillery (Québec) G1T $1 X_{4}$

Tél. : (418) 654-0259

Lieu historique national Cartier-Brébeuf

175, rue de l'Espinay, Case postale 2474

Terminus postal (Québee) G1K 7R3

Tél. : (418) 648-4038

Télec. : (418) 648-4825

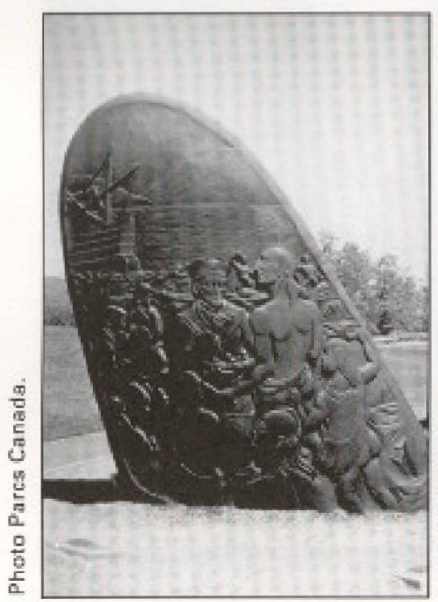

Rencontre entre Jacques Cartier et les autochtones : monument commémoratif.

\section{Québec (banlieue et région)}

Musée Arouănne

10, rue Chef-Alexandre-Duchesneau

Wendake (Québec) GOA 4V0

Túl. : (418) 845-1241

Village ancestral Huron

545 , rue Stanislas Koska

Wendake (Québec) G0A 4V0

Tél. : (418) 842-4308

\section{Cote Nord}

Musée amérindien et inuit de Godbout

134. chemin Pascal-Comeau

Godbout (Québec) GOH 1 Go

Tél. : (418) 567-7724

Musée régional de la Côte-Nord

500 , boulevard Laure. Case postale 725

Sept-îles (Québec)

Tél. : (418) 968-2070

Télec. : (418) 964-3213

Vieux Poste de Sept-Îles

99 , boulevard des Montagnais.

Case postale 725

Sept-lles (Québec) G4R 4K9

Tél. : (418) 968-2070

Télec. : (418) 964-3213

Chapelle indienne de Tadoussac

180 , rue de l'Église, Case postale 69

Tadoussac (Québec) GOT 2AO

Tél. (418) 235-4324

Shapituan

Musée innu de Uashat-Maliotenam

Sept-îles

(418) 962.4000

Archeo topo

Panorama préhistorique en

Haute-Cóte-Nord

498, rue de la Mer

Grande-Bergeronne (Québec) GoT 150

Tél. : (418) 232-6286 / (418) 2326695

\section{Gaspésie}

Musée de la Gaspésie

800 , boulevard Gaspé, Case postale 680

Gaspé (Québec) GOC 1 RO

Tél. : (418) 368-5710

Télec. : (418) 368.5715

Centre d'interprétation

de la Culture micmaque

2, rue Riverside Ouest

Restigouche Listuguj (Québec) GoC 2R0

Tél. : (418) 788-5034

Télec. : (418) 788-2058

\section{Saguenay-Lac-SaintJean}

Musée du Saguenay-Lac-Saint-Jean

534, rue Jacques-Cartier Est, Case postale 1415

Chicoutimi (Québec) G7H 5 K3

Tél. : (418) 545-9400

Télec. : (418) 545-9410
Centre d'interprétation de la Metabetchouane

243, rue Hébert

Desbiens (Québec) GoW 1No

T6l. : $(418) 346-5341$

Musée amérindien de Pointe-Bleue 407, rue Amishk

Mashteuiatsh (Québec) GoW $2 \mathrm{HO}$

Tél. : (418) 275-4842

Télec. : (418) 275-7494

Jardin zoologique de Saint-Félicien 2230, boulevard du Jardin. Case postale 90 Saint-Fülicien (Québec) G8K 2P8

Tál. : (418) 679-0543

Télec. : (418) 679-3647

\section{Abitibi-Témiscamingue}

Lieu historique national du Fort-Témiscamingue

Route 101, Case postale 636

Ville-Marie (Québec) JoZ 3W0

Tél. ; $(819)$ 629-3222

Wachiya, Centre d'interprétation

de la culture crie

145, avenue Perreault

Val d'Or (Québec) J9P 2G9

Mauricie-Bois-Franes

Musée des Abénaquis 108 , rue Waban-Aki Odanak (Québec) JOG $1 \mathrm{HO}$

Tél. : (514) 658-2600

Télec. : (514) 568-3553

Musée des arts et traditions populaires du Québec Section Préhistoire

2750, boulevard des Forges,

Case postale 1422

Trois-Rivières (Québec) G9A 5K2

Tél. : (819) 372-0406

Télec. : (819) 372-9907

\section{Hull-Ottawa}

Musée canadien des eivilisations 100, rue Laurier, Case postale 3100. Succursale B

Hull (Québec) J8X $4 \mathrm{H}_{2}$

Tél. : (819) 776-7000

Télec. ; (819) 776-8300

Musée des Beaux-Arts du Canada 380 , Promenade Sussex

Case postale 427 , Succursale A

Ottawa (Ontario) $\mathrm{K} 1 \mathrm{~N} 9 \mathrm{~N} 4$

Tél. : (613) 990-9204

Télec. : (613) 993-4385

\section{Nunavik, Nord du Québec}

Musée inuit de Inukjuak

Contacter : L'institut culturel Auataq Inukjuak (Québec) J0M $1 \mathrm{M0}$

Tél. : (819) 254-8919

A Montréal : (514) 637-9707 